\title{
Optimization of Features for Image Enhancement Using Local and Global Features
}

\author{
Amit Tiwari ${ }^{1}$, Akhilesh Upadhyay ${ }^{2}$ \\ ${ }^{1}$ Department of Electronics \& Communication, SIRT College Bhopal (M.P) India \\ ${ }^{2}$ Professor, Department of Electronics \& Communication, SIRT College Bhopal (M.P) India
}

\begin{abstract}
Image enhancement is used to improve the digital quality of image. It is used to improve the poor quality of image that is too used to improve bad quality of picture into good picture or image. Contrast enhancement is acquiring clear image through brightness intensity value redistribution. For the enhancement of image used feature optimization technique. The feature optimization technique used particle of swarm optimization for the selection of local and global feature of image. The optimized feature is distributing overall image and the quality of image enhanced. The experimental results show the mean with the traditional enhancement methods, the proposed threshold-based enhancement digital image enhancement algorithm for mixed digital image enhancement is relatively clear, especially in the more damage pixel, more complex cases", can show its good performance.
\end{abstract}

Keywords: LGE, PSO, BH, CH, SNR.

\section{Introduction}

Image detail upgrade calculations can increment visual appearance of pictures. They upgrade fine subtle elements while maintain a strategic distance from radiance antiques and inclination inversion ancient rarities around edges. The detail improvement procedure is a generally utilized picture altering device. Existing subtle element improvement calculations depend nervous protecting disintegration calculations. A source picture is first decayed into a base layer which is framed by homogeneous locales with sharp edges and a detail layer which is made out of fine points of interest or surfaces by means of the edge-safeguarding disintegration calculation, then a detail-upgraded picture is created by increasing the detail layer [2]. Picture improvement is a procedure which changes the pixel's force of the information picture in order to improve the yield picture looks. Picture improvement method has been proposed in numerous use of picture preparing where subjective nature of picture is most essential and target nature of picture is relied on upon application conditions. Picture improvement is the procedure of remapping the first picture pixels into a picture, a remarkable same, however decorated with more differentiation - in which, the points of interest parade more than what they were in the first picture. Picture improvement strategies are valuable for their tasteful perspectives as well as help in picture division, picture investigation, include extraction, and so on. As indicated by down to earth utilize, picture improvement strategies must satisfy particular requests in numerous applications, for example, photography, protest location and acknowledgment, optical character acknowledgment (OCR), and particularly in restorative picture applications e.g. mammographic picture upgrade and denoising which will enhance the conclusion method in the early bosom growth recognition. What's more, the term picture improvement is turned out, in its own particular nature - pay to the likely disadvantages created by wrong lighting amid the photograph shoot. Numerous applications may have a tendency to flop because of mid nature of the picture e.g. dull pictures with low SNR, or regardless in which the picture histogram has an accentuation on one specific territory [2].

The crucial upgrade required in mammography is an expansion conversely. Differentiate between threatening tissue and nor-mal thick tissue might be available on a mammogram, however beneath the limit of human discernment. Their accentuation at this stage is to furnish the radiologist with an unrivaled picture. Before, a few picture differentiate upgrade techniques have been proposed. Numerous picture improvement methodologies were proposed in the writing. These upgrade strategies are not specifically reasonable for mammogram pictures. As the mammogram pictures are surface in nature, it is extremely hard to get appropriate improvement for these pictures by these routine strategies.

Versatile un-sharp veiling procedure was likewise connected for picture differentiates upgrade. It is additionally ailing in recognizing low complexity edges like miniaturized scale calcification display in the mammogram picture, when it is connected to the mammogram picture. The exploration works have been done on mammograms for its differentiation upgrade and for ID of picture components like group of small scale calcification and masses connected with bosom growth. These strategies presented upgrade on mammogram highlights utilizing versatile neighborhood technique which are additionally not invulnerable to commotion and delivers more ancient rarities. Rangayyan et al. worked in mammographic picture differentiate upgrade in which the complexity has been enhanced while trading off the expectation of the first picture. They broke down the adequacy of their versatile neighborhood differentiate upgrade (ANCE) method in expanding the affectability of bosom malignancy determination. An option method for mammogram differentiate upgrade utilizing wavelet based techniques came about as a part of change in saving the points of interest in the picture at the cost of clamor enhancement [10]. 


\section{International Journal of Science and Research (IJSR) \\ ISSN (Online): 2319-7064}

Index Copernicus Value (2015): 78.96 | Impact Factor (2015): 6.391

\section{Edge-Preserving}

Picture edge-safeguarding deterioration calculations can be di-vided into two classifications: neighborhood filter based and worldwide improvement based. Middle filter, a notable denoise filter; can be utilized as an edge-saving decay filter. An iterative middle filter was utilized as an edge-pre-serving decay instrument in a summed up un-sharp concealing calculation. Two-sided filtering (BF) joins a range filter with an area filter to safeguard edges. It is a basic and broadly utilized nearby weighted normal filter, however it might display slope inversion ancient rarities close to a few edges when utilized for detail upgrade. A guided picture filter (GIF) got from a nearby straight model can dodge the angle inversion relics, in this way it out-plays out the BF. The computational cost of all the neighborhood filters is low. Nonetheless they experience the ill effects of coronas close to a few edges. This issue can be overcome by utilizing worldwide enhancement based filters. The aggregate variety filter utilizes a standard based regularization term to evacuate clamors in pictures, which is likewise considered as an edge-safeguarding decay calculation. Weighted Least Squares (WLS) based multi-scale decay calculation breaks down a picture to two layers by understanding a weighted minimum square enhancement issue. A quickened iterative shrinkage calculation is proposed to deteriorate and improve picture. Inadequate models normally can give better result in picture handling calculations. The standard of the slope of picture was utilized as a part of the smoothing term. It can safeguard edges superior to WLS [2]. In fidelity is utilized as a part of the plan rather than fidelity which was utilized as a part of [9].

\section{Problem Formulation}

The basic idea behind this thesis is the estimation of the uncorrupted image from the distorted or noisy image, and is also referred to as image "enhancement". There are various methods to help restore an image from noisy distortions. Selecting the appropriate method plays a major role in getting the desired image. The enhancement methods tend to be problem specific. For example, a method that is used to denoise. Satellite images may not be suitable for enhancement medical images. Each method is compared and classified in terms of its efficiency. In order to quantify the performance of the various enhancement algorithms, a highquality image is taken and some known noise is added to it. This would then be given as input to the enhancement algorithm, which produces an image close to the original high quality image. The performance of each algorithm is compared by computing Signal to Noise Ratio (SNR) besides the visual interpretation. Also, we find in general problem in image denoising process used wavelet transform and artificial neural network model.

- The mean template approach: The original gray value of one pixel and its surrounding neighbouring pixel gray value are divided by the sum of these pixels, the average value will be the gray value of the corresponding pixel of new image. This method has the advantage: not only easy to understand, and computation easy, suitable for small image and noise less situation. But when the image is larger and more noise, the use of the mean template and cannot effectively remove the noise, and the average operation, will have some degree of blurred images'".

- The neighborhood smoothing method: Using the average gray value of the pixel and its neighborhood look upon as the gray value of the pixel, this method is simple, but it will make the image blurred boundaries. Therefore, in order to better image enhancement. After some research enhancement algorithm. Proposed a threshold based on digital image enhancement hybrid algorithms. It has several features:

- LGE function not distribute lower pixel content channel.

- Very difficult to collect lower content of image using channel distribution.

\section{Proposed Algorithm}

In this paper proposed image enhancement technique based on Particle swarm optimization. Particle swarm optimization is dynamic population based optimization technique. In this technique, all pixel value treats as particle and measure the similarity of pixel value based on the velocity of difference value. If the value of difference value is 0 treat as standard data and the value of difference value is not 0 treat as noise and degraded part of image. In the continuity of chapter discuss the LGE algorithm of image enhancement, particle swarm optimization, proposed algorithm and proposed model.

In this section discuss image enhancement methodology based on LGE function and particle of swarm optimization. The LGE function distributes the all data in the form of channel in terms of matrix. The image features are extracted from the image using LGE function. PSO acts as a clustering mechanism that projects $\mathrm{N}$-dimensional features from the LGE function into an M-dimensional feature space. The resulting vectors are fed into a PSO that categorizes them onto one of the relearned damage pixel classes. The proposed scheme is work along with particle swarm algorithm. The particle swarm algorithm process the collection task of local intensity of image data. The collected damage pixel value combined with high intensity image value and generates vector value for the process. They mapped features from each frame of the word onto the PSO output to form a trajectory of winner nodes for a given word. The PSO learns this trajectory for each damage pixel constraints value is comprised of a hierarchical organization of PSO and PSO. PSO receives inputs from the LGE function bank and maps onto an M-dimensional space where $\mathrm{M}$ is the dimensionality of the PSO output node distribution. The transformed feature vectors are fed into the PSO, which classifies them. We call the feature space generated from the LGE function output as primary feature space and $\mathrm{M}$ dimensional feature space from PSO output as secondary feature space. The vectors from the secondary feature space are called secondary feature vectors.

\subsection{Processing of Proposed Algorithm}

Step1. Initially input image passes through LGE function and decomposed into channel

Step2.the all channel measure different intensity value.

\section{Volume 6 Issue 7, July 2017 www.ijsr.net}




\section{International Journal of Science and Research (IJSR) \\ ISSN (Online): 2319-7064}

Index Copernicus Value (2015): 78.96 | Impact Factor (2015): 6.391

Step3. The collection of lower intensity value used particle of swarm algorithm

step4. Particle swarm algorithm collects the local damage pixel value after that combined with high intensity value.

Step5. After collecting total damage pixel value convert into feature vector image data passes through self-filter

Step6. In phase of feature mapping in feature space of PSO network create a fixed cluster according to threshold of details of image part.

Step7. Here show steps of processing of PSO algorithm

1) Initialize each pixel as particle

2) Choose a random vector from to the PSO.

3) Measure the velocity of difference in two particles.

4) The particle of the neighborhood around the Gbest is calculated. The size of the neighborhood decreases with each iteration.

5) Repeat from step 2 for enough iteration for convergence.

1) Calculating the Pbest is done according to the Euclidean distance among the pixel.

2) This gives a good measurement of how similar the two sets of data are to each other.

Steps 8. After processing of PSO out data of image is also passes through

Step 9. Finally gets enhanced image and measure value of PSNR.

\subsection{Proposed Model}

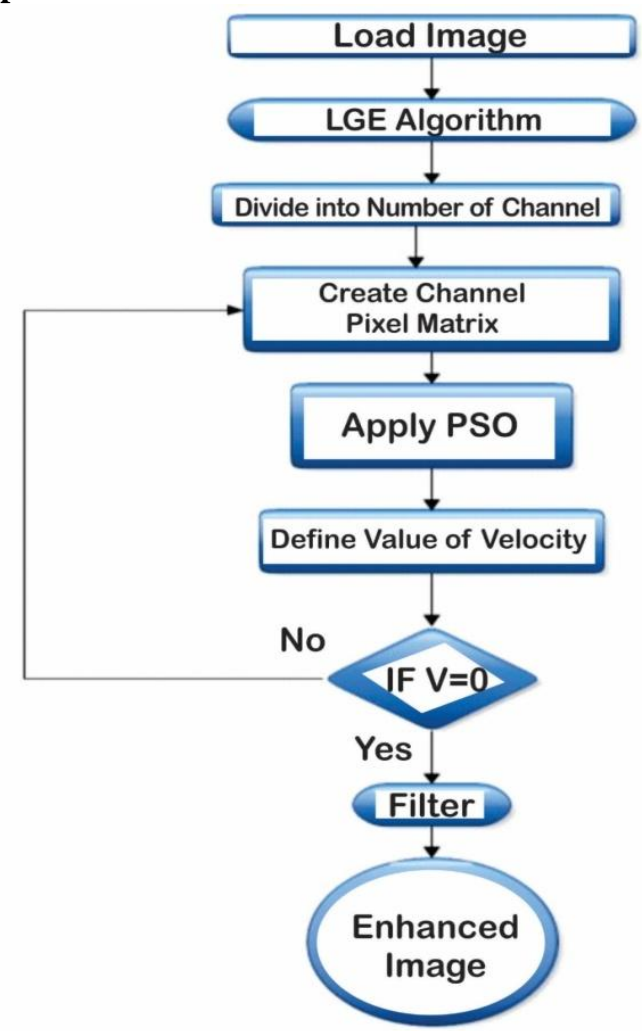

Figure 1: Proposed Model of our Algorithm

\section{Experimental Result Analysis}

To evaluate the performance of proposed method of image enhancement based on the optimization of global and local features using particle swarm optimization. we have use MATLAB software 7.8.0 with a variety of image dataset used for experimental task.

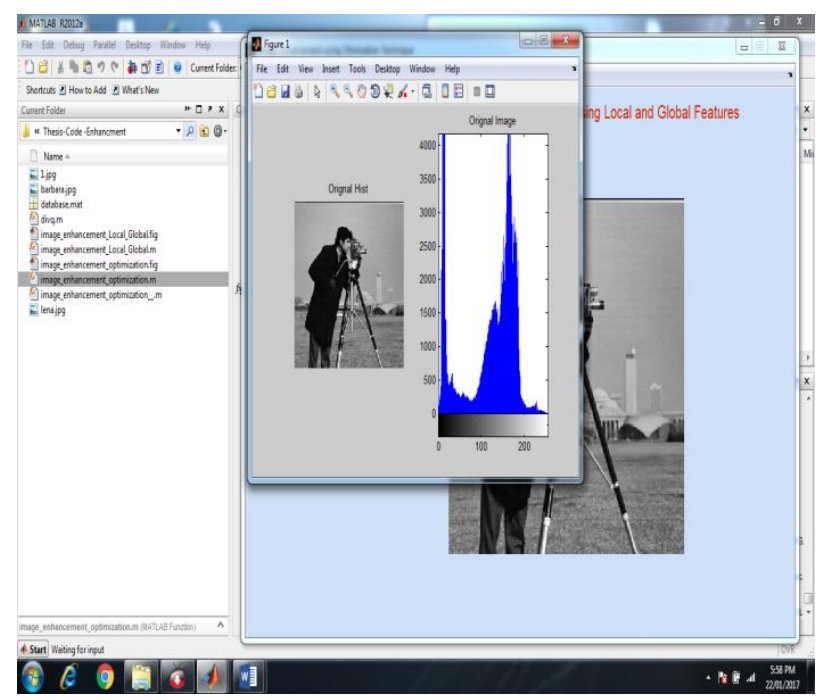

Figure 2: implementation window represented that output window when click on PROPOSED-Method button in Optimization of Features for Image Enhancement using local and Global Features.

Table 1: after the implementation, we get comparative result in load a 1.jpeg image using LGE-method and PROPOSEDmethod in Optimization of Features for Image Enhancement using local and Global Features.

\begin{tabular}{|c|c|c|}
\hline \multicolumn{3}{|c|}{ Imported Image -1} \\
\hline Method & $\mathrm{BH}$ & $\mathrm{CH}$ \\
\hline LGE & 31.8267 & 18.7238 \\
\hline Proposed & 13.3715 & 34.0655 \\
\hline
\end{tabular}

Table 2: after the implementation, we get comparative result in load a barbara.jpeg image using LGE-method and PROPOSED-method in Optimization of Features for Image Enhancement using local and Global Features.

\begin{tabular}{|c|c|c|}
\hline \multicolumn{3}{|c|}{ Imported Image - Barbara } \\
\hline Method & BH & CH \\
\hline LGE & 24.405 & 14.3575 \\
\hline Proposed & 10.2534 & 26.1217 \\
\hline
\end{tabular}

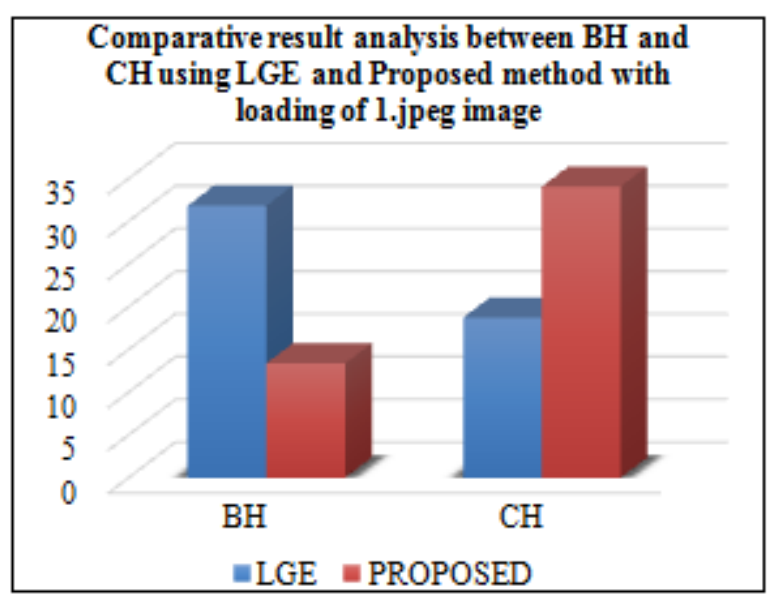

Figure 3: Given graph represented that comparative result (BH and $\mathrm{CH}$ ) with load a 1.jpeg image using LGE-method and PROPOSED-method in Optimization of Features for Image Enhancement using local and Global Features. 


\section{International Journal of Science and Research (IJSR)}

ISSN (Online): 2319-7064

Index Copernicus Value (2015): 78.96 | Impact Factor (2015): 6.391

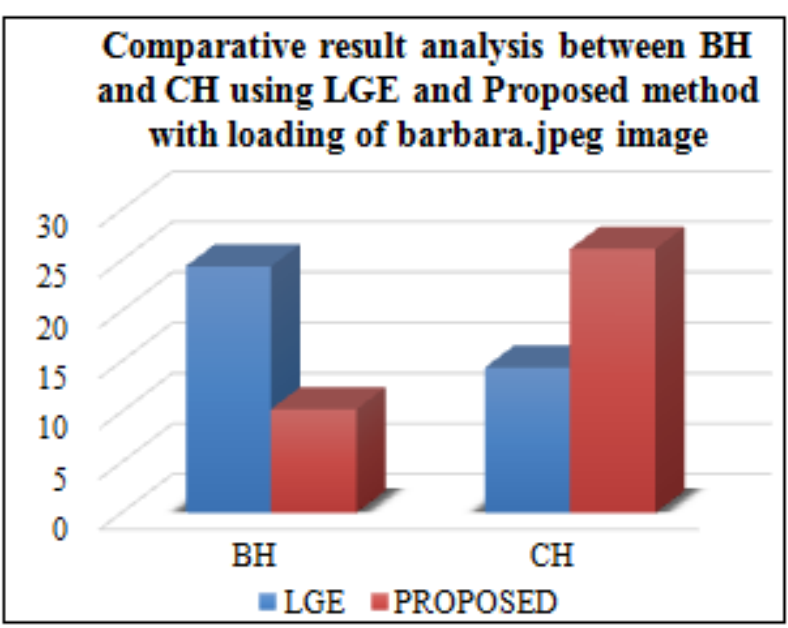

Figure 4: Given graph represented that comparative result (BH and $\mathrm{CH}$ ) with load a barbara.jpeg image using LGEmethod and PROPOSED-method in Optimization of

Features for Image Enhancement using local and Global Features.

\section{Conclusion and Future Work}

In this paper, an image enhancement technique using LGE and POS method based on channel filtration technique for image enhancement. PSO were used to find correlation between damage pixel and original channel coefficients. Experimental results showed capability of proposed method to remove damage pixel in terms of $\mathrm{BH}$ and $\mathrm{CH}$. In this paper, we proposed a hybrid method for image enhancement for normal image. Our experimental result shows that better result in compression of old and traditional method of image enhancement. But the computational time of process is increase. In future, we used optimizations method for the reduction of time and improvement of quality of image.

\section{References}

[1] Sampada S Pathak, Prashant Dahiwale and Ganesh Padole "A Combined Effect of Local and Global Method for Contrast Image Enhancement”, IEEE, 2015, Pp 1-5.

[2] Fei Kou, Weihai Chen, Zhengguo Li and Changyun Wen "Content Adaptive Image Detail Enhancement", IEEE, 2015, Pp 211-215.

[3] Wei Fan, Kai Wang, François Cayre and Zhang Xiong "Median Filtered Image Quality Enhancement and AntiForensics via Variational Deconvolution", IEEE, 2015, Pp 1076-1091.

[4] Saber Yaghoobi, Saeed Hemayat and HamedMojallali "Image Gray-level Enhancement using Black Hole Algorithm”, RPIRA, 2015, Pp 1-5.

[5] Xueyang Fu, Yinghao Liao, Delu Zeng, Yue Huang, Xiao-Ping Zhang and Xinghao Ding "A Probabilistic Method for Image Enhancement With Simultaneous Illumination and Reflectance Estimation", IEEE, 2015, Pp 4965-4977.

[6] Ms. NishatFatema and Mr. M.S. Chaudhari "Survey on Digital Image Forensics Using Different Contrast Enhancement Techniques", IJARCCE, 2016, Pp 627629.
[7] Raman Maini and Himanshu Aggarwal "A Comprehensive Review of Image Enhancement Techniques", JOURNAL OF COMPUTING, 2010, Pp 8-13.

[8] TurgayCelik and TardiTjahjadi "Automatic Image Equalization and Contrast Automatic Image Equalization and Contrast", IEEE, 2012, Pp 1-21.

[9] N. Sridevi and Mrs.S. Usha Rani "AN IMPROVED IMAGE QUALITY ENHANCEMENT AND ANTIFORENSICS BASED ON EDGE PRESERVING FILTER", IJIRSE, 2016, Pp 237-243.

[10] M. Sundaram, K. Ramar, N. Arumugam and G. Prabin "Histogram Modified Local Contrast Enhancement for mammogram images", Elsevier, 2011, Pp 5809-5816.

[11] MarivíTello Alonso, Carlos López-Martínez, Jordi J. Mallorquí and Philippe Salembier "Edge Enhancement Algorithm Based on the Wavelet Transform for Automatic Edge Detection in SAR Images", IEEE, 2011, Pp 222-235.

[12] Carsten Gottschlich "Curved Gabor Filters for Fingerprint Image Enhancement", arXiv, 2014, Pp 1-18.

[13]Ja-Hwung Su, Wei-Jyun Huang, Philip S. Yu and Vincent S. Tseng "Efficient Relevance Feedback for Content-based Image Retrieval by Mining User Navigation Patterns", IEEE, 2010, Pp 1-14.

[14]Hasan Demirel, CagriOzcinar and GholamrezaAnbarjafari "Satellite Image Contrast Enhancement Using Discrete Wavelet Transform and Singular Value Decomposition", IEEE, 2010, Pp 1-5.

[15] PranaliTurkhade and Prof. U.W. Hore "Analysis for Image Quality Improvement using Denoise Filtering Techniques", IJARCCE, 2016, Pp 423-428.

[16] Yinghua Li, Tian Pu, Jian Cheng,"A BIOLOGICALL Y INSPIRED NEURAL NETWORK FOR IMAGE ENHANCEMENT",2010 International Symposium on Intelligent Signal Processing and Communication Systems (ISPACS 201O) December 6-8, 2010

[17] Seyed Mohammad Entezarmahdi and Mehran Yazdi, "Stationary Image Resolution Enhancement on the Basis of Contourlet and Wavelet Transforms by means of the Artificial Neural Network", IEEE, 2010.

[18] G.G. Bhutada R.S. Anand S.C. Saxena, "Image enhancement by wavelet-based thresholding neural network with adaptive learning rate", IET Image Process., 2011, Vol. 5, Iss. 7, pp. 573-582.

[19]Jingchao ZHAO, Shiru QU, "The Fuzzy Nonlinear Enhancement Algorithm of Infrared Image Based on Curvelet Transform", Elsevier Ltd, 2011, Pp 3754 3758.

[20] Changyan Xiao, Marius Staring, Denis Shamonin, Johan H.C. Reiber, Jan Stolk, Berend. Stoel "A strain energy filter for 3D vessel enhancement with application to pulmonary CT images", Elsevier Ltd, 2011, 112-124. 